\title{
Looking beyond Lucentis on the management of macular degeneration
}

\begin{abstract}
Purpose Age-related macular degeneration (AMD) is the leading cause of blindness in the developed world. In the past decade, AMD research has improved our understanding of the pathogenesis of the condition. There is no doubt that anti-VEGF therapies will play a major role in the reduction of blindness, but it needs close monitoring and frequent treatments. In this review we summarise the key pathophysiology of the condition and some of the new treatment strategies under development.

Methods Literature review.

Results Oxidative stress, inflammation, hypoxia, and angiogenesis are key pathophysiological processes in AMD, this diversity has provided a variety of possible areas amenable to modification to enhance the treatment options for AMD.

Eye (2008) 22, 742-750; doi:10.1038/sj.eye.6703008; published online 11 January 2008
\end{abstract}

Keywords: age-related macular degeneration; oxidative stress; protein kinase inhibitors; angiogenesis; siRNA; VEGF trap

\section{Oxford, UK}

Correspondence: $\vee$ Chong, Consultant Ophthalmic Surgeon, Oxford Eye Hospital, Headley Road, Headington, Oxford OX3 9DU, UK

Tel: + 4401865 234736;

Fax: +440870 7454778 .

E-mail: victor@

eretina.org

Received: 14 September 2007

Accepted: 14 September 2007

Published online: 11 January 2008
EC Fletcher and NV Chong

uptake rate, and significant confusion.

Visudyne ${ }^{\mathbb{R}}$ and Macugen ${ }^{\mathbb{R}}$ can stabilise vision, while Lucentis ${ }^{\circledR}$ can improve vision, however, the treatment frequency and the intensity of the follow-up as well as the cost of the drugs are significant limiting factors to reducing blindness. Furthermore, it is becoming clear that late presentation is more likely to lead to blindness than early presentation; and evidence is emerging that final visual acuity is closely related to the presenting visual acuity. ${ }^{7,8}$

In this review, we are going to examine some of the treatment strategies that might be able to reduce AMD blindness; some of these strategies can be implemented today, while many are hypothetical based on current laboratory data and ongoing clinical trials.

AMD is a systemic disease

The most important research findings in AMD over the past decade include anti-VEGF therapies, the identification of the several genetic factors and the appreciation of the importance of smoking, and systemic biomarkers such as C-reactive protein (CRP). However, none of the major 'at risk' genetic factors in AMD identified so far are eye specific, let alone macular specific. So, it is difficult to appreciate how these non-specific factors cause a very specific localised macular disease.

For many, AMD is a macular disease, it is easy to appreciate why, as the name suggests most of the clinically visible features are in the macular region. However, if one examines donor eyes of patients with AMD, the pathology in the macula extends over the entire retina. In fact, the peripheral retina often has more significant pathology but invasion of choroidal neovascularisation (CNV) into the subretinal space in this location is very rare. So the clinical observation is not due to presentation bias, but 
due more to the vulnerable macula, making it a target for the disease process.

Many anatomical and biological features separate the macula from the peripheral retina. For instance, we have previously published that the elastic layer of the Bruch's membrane in the macula is significantly thinner and more porous than the peripheral retina, this was more pronounced in AMD donors, ${ }^{9}$ which may be the factor which allows $\mathrm{CNV}$ invasion. So the concept of the more vulnerable site and the greater chance of disease, rather than specific targeting is important.

To explain the recent findings that both genetic predisposition and environmental factors are important in the pathogenesis of AMD, we would have to extend the above concept that AMD is in fact a systemic disease to include the fact that the eye is more vulnerable and hence only the eye shows significant disease. The close relationship in terms of shared risk factors of AMD and atherosclerosis further support the argument that Bruch's membrane is, in fact, a specialised blood vessel wall. ${ }^{10}$ So it is logical to accept that atherosclerosis research may one day benefit AMD patients.

Once this concept is accepted, prevention strategies can then be developed based on systemic measurement and systemic modification. Before looking at the different strategies, we are going to summarise some of the important proposed pathogenic mechanisms of AMD, which might allow us to target treatment.

\section{Oxidative stress}

Prolonged and repeated exposure to oxidants is a feature of many chronic age-related diseases such as neoplasia, atherosclerosis, and neurodegenerative disease such as Alzheimer's as well as AMD. ${ }^{11}$ The macula is particularly prone to oxidative stress due to the high oxygen tension, high levels of polyunsaturated fats, chronic light exposure, and close contact with oxidative molecules and lipofuscin, a secondary photosensitising agent, which accumulates with aging.

Oxidants and free radicals are a normal by-product of physiological processes. Oxidative stress only develops, when there is a shift in the balance of equilibrium in favour of the oxidative components. ${ }^{12}$ Oxidant exposure via oxygen metabolism in aerobic respiration is due to the generation of a small quantity of reactive oxygen species (ROS). These include superoxide anion, hydroxyl radical, and hydrogen peroxide (Figures 1 and 2). ROS impair cell function by attacking lipid membranes (lipid peroxidation), as well as damage to surface proteins, DNA, and transmembrane glycoproteins. The eye, in particular the macular region, has a highly concentrated area of polyunsaturated fatty acids, which due to the high number of double bonds are very

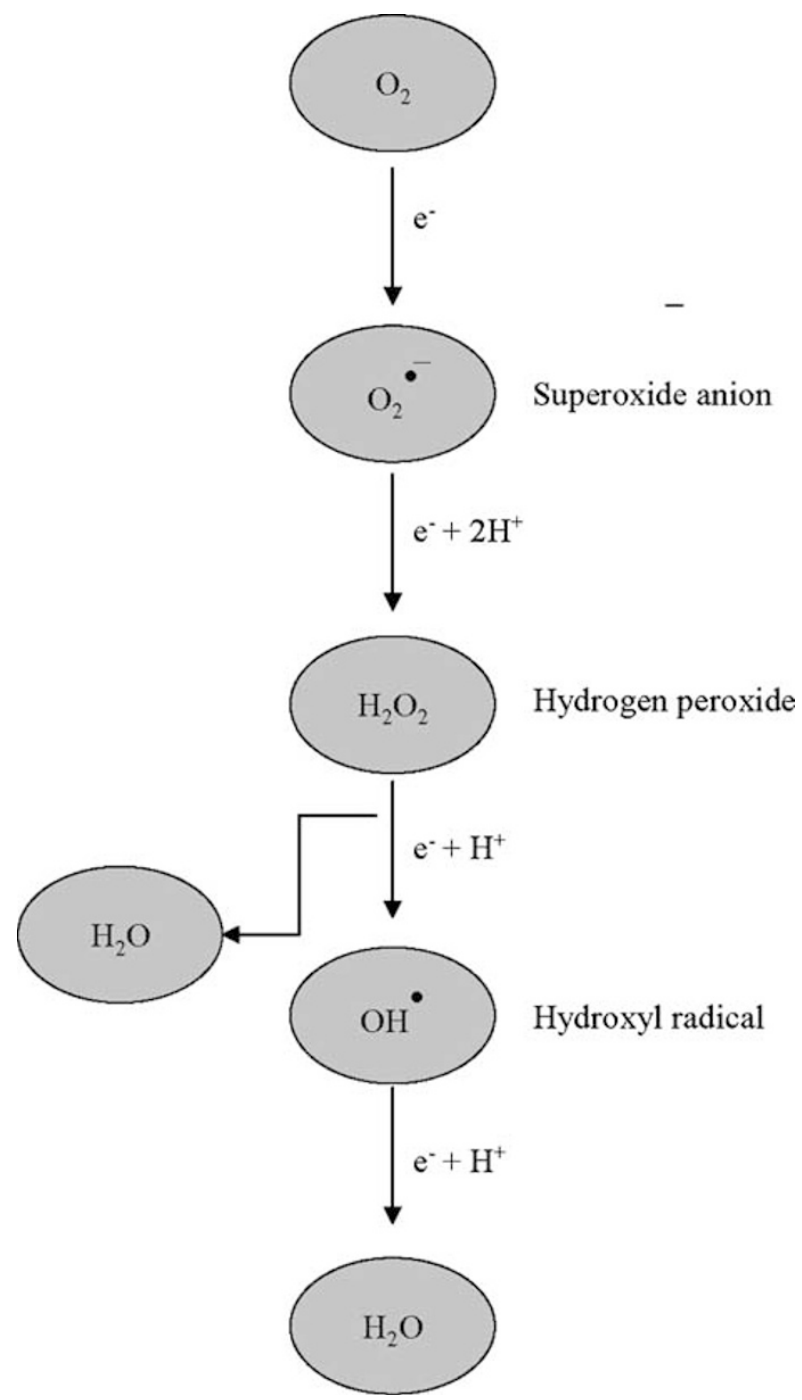

Figure 1 Generation of reactive oxygen species.

susceptible to oxidation. There are also increased levels of oxidised phospholipids in the photoreceptors and the RPE cells of the aging macula and this is further increased in eyes with AMD. ${ }^{11}$

Exposure to radiation, such as sunlight or in particular blue light produces singlet oxygen due to photosensitisation. The amount and type of free radical formation is dependant upon the wavelength of light. Singlet oxygen can then attack membranes and other cell components especially of the photoreceptor outer segments.

Photoreceptor turnover involves ingestion of outer segments by the RPE through its apical surface in the form of phagosomes, which in turn are broken down by lysosomes. The degraded material is then exocytosed on the basal side. Accumulation of oxidised photoreceptor outer segments inhibits the function of phagosomes and so there is incomplete breakdown of the outer segment 


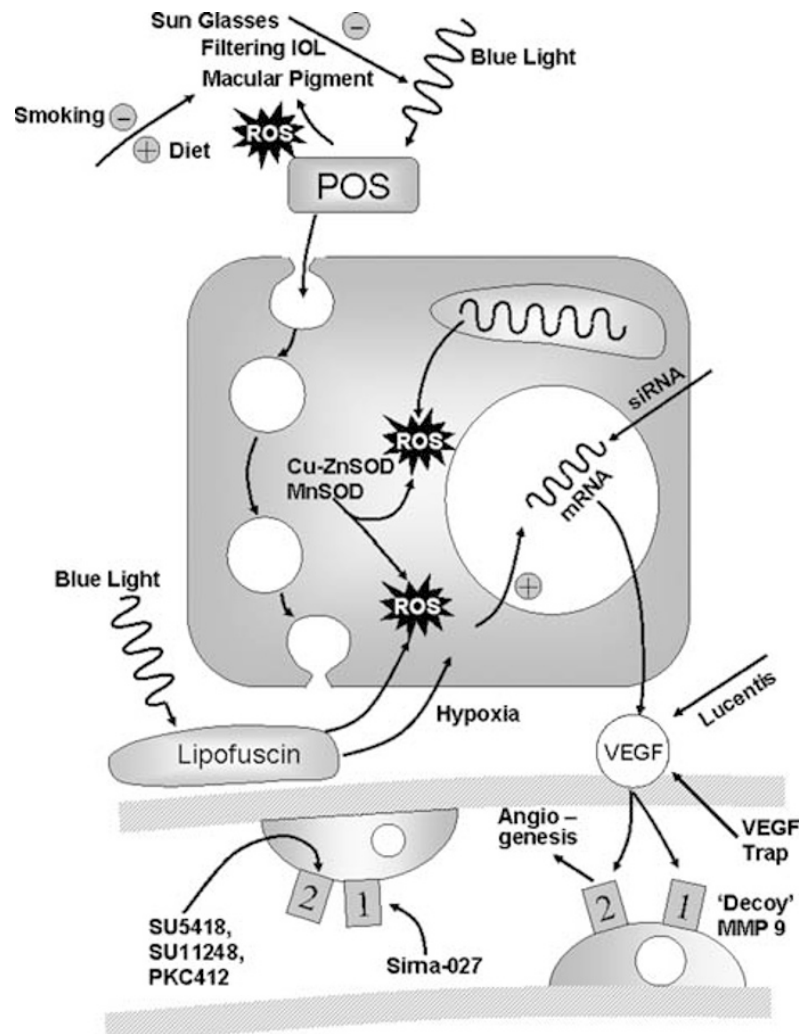

Figure 2 Diagram to illustrate site of action of reactive oxygen species and treatment strategies.

discs which then increase liposfuscin formation. ${ }^{13,14}$ The association between sunlight exposure and AMD is however difficult to determine as the exposure is chronic in nature and difficult to measure and quantify. ${ }^{15}$ Hence, there is only weak epidemiologic evidence that excessive exposure to light is associated with AMD. ${ }^{16,17}$

Furthermore, chronic excessive exposure to light increases the risk of cataract formation, ${ }^{18}$ this might be a form of natural protection for the retina. With the advances in cataract surgery, surgery is often preformed at an earlier age. Although, it remains controversial whether cataract surgery increases the rate of progression of AMD, it appears not to be the case in the short to medium term. It is more difficult to be certain for the long term (more than 5 years), and for those who are already at risk with AMD (either genetically or otherwise).

\section{Compartmentalisation}

Formation of a less harmful product or diversion of the oxidant away from areas in which it could potentially do harm is a further mechanism of antioxidation. The RPE has endogenous mechanisms for antioxidation in the form of enzymatic cleavage of the ROS with superoxide dismutases; copper-zinc superoxide dismutase $(\mathrm{Cu}-\mathrm{Zn}$ SOD) or mitochondrial manganese superoxide dismutase (Mn SOD) to form a less harmful product. A direct correlation with upregulation of this antioxidant pathway is seen with the different stages of AMD. ${ }^{18}$ There is currently no therapeutic application for the enzymes themselves, however, they are reliant upon enzyme cofactors such as zinc and copper.

The macular pigment (MP) antioxidant function is to quench singlet oxygen and scavenge $\mathrm{ROS}$ via its $\mathrm{OH}$ functional group, which makes it susceptible to oxidation. ${ }^{19}$ This slows the peroxidation of the membrane phospholipids. In addition, the double bonds on the polyene chain are preferentially oxidised thus limiting damage to the other cell structures.

Other free radical scavengers include $\beta$-carotene ${ }^{13}$ and vitamin $C$, and E. Dietary antioxidant supplementation of the AREDS formula which includes high intake of Vitamin C and E, Zinc, Copper, and $\beta$ - carotene, has been shown to reduce the progression of AMD by $25 \%$ in category three and $48 \%$ in category four. ${ }^{4}$

\section{Smoking}

There is reproducible evidence of a consistent association between smoking and AMD. ${ }^{20,21}$ Analysis of pooled data from three population-based cohort studies showed that the relative risk of late AMD is 2.4 among current smokers as compared with those who never smoked, with an insignificant risk of 1.29 among former smokers. ${ }^{22}$ However, current smokers who are homozygous for the complement factor $\mathrm{H}(\mathrm{CFH}) \mathrm{Y} 402 \mathrm{H}$ polymorphism have a relative risk of 34.0 for late AMD as compared with non-smokers who do not have this polymorphism. ${ }^{23}$ The risk is 7.6 for non-smokers who are homozygous for the CFH Y402H polymorphism. ${ }^{23}$

Smoking is a pro-oxidative factor, ${ }^{24}$ and it is associated with a lower level of macular pigment (MP) optical density (infra vide). ${ }^{25,26}$ Plasma nicotine activates retinal phospholipase A2, leading to the formation of arachidonic acid, a precursor of prostaglandins, and various cytokines, which are inflammatory mediators. ${ }^{27}$ Tar from cigarettes also contains a high concentration of a pro-oxidant hydroquinone. Lesions similar to those in the aging retina develop in the eyes of mice that are exposed to cigarette smoke or dietary hydroquinone. ${ }^{28}$

\section{Macular pigments}

Carotenoids were initially discovered as pigments in plants, which have the ability to absorb visible light. The macula selectively accumulates two carotenoids, which form the MP providing the yellow colouration seen on biomicroscopy. MP consists of two xanthophylls; lutein, 
and zeaxanthine with the latter predominantly found in the foveal region and the former in the parafoveal region.

The MP have an absorption peak at $460 \mathrm{~nm}$ of visible light and therefore reduce the exposure of the photoreceptor outer segments to blue light phototoxicity by $40-90 \% .^{29}$ Due to orientation of lutein in the retina is able to absorb blue light from all directions thus conferring more protection than zeaxanthine which is orientated in only one direction. ${ }^{19}$

Reduced levels of MP are thought to be independently associated with smoking, however the mechanism is unknown. Recent observations show MP levels in clinically normal individuals to be lower in smokers and those with family history of AMD. ${ }^{25}$ There are at least two possibilities to explain this:

(1) Smokers have an excessive oxidative state, RPE cells have an increased demand for anti-oxidants, such as MP, so less MP can be transported into the retina. The retina is not protected and this, in turn, leads to AMD.

(2) Smokers have excessive oxidative state, this leads to AMD and sick RPE cells. As the cells are sick, they cannot transport MP to the retina. MP in retina is not associated with AMD but just a sign of sick RPE cells.

Nonetheless, it remains controversial whether dietary supplementation of MP is beneficial in AMD. ${ }^{25,30,31} \mathrm{MP}$ levels in the retina can be increased by dietary supplementation $^{32,33}$ and as the MP level in the retina varies a lot in the population, it might be reasonable to supplement only those with low MP levels.

There is a small study, which suggests Lutein supplements can improve vision in the short term, ${ }^{34}$ adding to the concern surrounding $\beta$-carotene in the AREDS preparation, this has lead to significant confusion.

\section{AMD prevention strategies based on the reduction of oxidative stress}

\section{Local strategies}

The ability to prevent the formation of excess oxidants is the primary step in anti-oxidation. Prevention from light exposure includes eye protection such as sunglasses or specialised intraocular lenses. The cornea is able to filter out short wavelength light (below $300 \mathrm{~nm}$ ) with the crystalline lens providing further filtering (300-400 nm). Intraocular implant (IOL) contains a UV-blocking filter, however, suggested phototoxicity from the blue light in the visible spectrum has prompted the development of blue light filtering (yellow-tinted) IOL. Blue blocking IOL can only block about $20 \%$ of blue light as compared to the natural crystalline lens and less than $50 \%$ of sunglasses. ${ }^{35}$ There is also a concern regarding sleep patterns due to the alteration of the saccadic rhythm in the use of these yellow IOLs. This regards however the reduction of improvement of sleep pattern after the surgery, therefore if there was no pre-operative sleep disorder, it may not matter. Contrast sensitivity does not appear to be affected, and in fact, it might be slightly enhanced by these IOLs. None of these measures can be easily studied by randomised controlled trials, so it might be sensible to use sunglasses in bright light, and use yellow tinted IOL if having surgery at an early age or in patients at risk of developing end-staged AMD.

\section{Systemic strategies}

Being a non-smoker and having a high serum level of anti-oxidants can significantly reduce the risk of AMD in $10 \%$ of the population, for those with the 'at risk' genetic polymorphisms. It is debatable whether genetic testing is justified at the moment, as one could argue that no one should smoke and everyone should have a balanced diet rich in anti-oxidants. However, genetic testing might enhance motivation. Nonetheless, negative results might give false assurance, as our understanding of AMD genetics is still limited.

AREDS formula can reduce progression risk significantly in those with Category 3 (large drusen or atrophy or significant intermediate drusen in both eyes) and 4 (reduced vision in one eye due to AMD). It is important for ophthalmic professions to recommend AREDS formula to these patients. It is also important for ophthalmic professions to understand the difference between the different preparations of 'eye vitamins'. The public is confused due to the numerous preparations; the professionals should be clear about the evidence behind their recommendation. Any preparations other than AREDS at the moment, could not be considered to be evidence based. Photographic screening of early asymptomatic AMD like those of diabetic retinopathy screening is currently not considered to be cost-effective. However, those at high risks, that is smoker, older age, family history (or genetic tested) individuals, screening might be useful. The screening is unlikely to be annual, the exact screening strategy remains to be studied.

It is even more controversial on the recommendation of MP supplementation, such as lutein and zeaxanthine.

At the moment, the evidence is limited and theoretical at best. The widespread use of lutein in 'smoker preparation' is worrying, due to the similarity of the molecular structure of lutein and $\beta$-carotene, if the latter increases the risk of lung cancer in smokers, the former might do so too. So for smoker, stop smoking might be more important than anything else. If MP supplement is suggested for non-smoker, it might be sensible to recommend only to those who have low level of MP in 
the retina. This can be measured relatively easily by commercially available machine such as the Maculoscope ${ }^{\mathbb{R}}$. AREDS II is a new study supported by the US National Eye Institute; in this new study, lutein will be studied in detail, so we might have a better idea in about 10 years time. Until then, this will remain controversial. Another supplement docosahexanoic acid, an Omega-3 fatty acid, which stabilises biophysiological properties of membranes such as the photoreceptor outer segments will also be studied in AREDS II.

\section{Inflammation and AMD}

Inflammation and the immune system are increasingly seen to play a significant role in the pathogenesis of AMD. The hallmark of AMD was initially seen as the build up of drusen, which are thought to be induced by immune-mediated events. More recently with the identification of the role of $\mathrm{CFH}$ and more recently $\mathrm{C} 3$ in the genetic pathway of AMD has helped to confirm this association.

The complement system is part of the innate immune response associated with three different pathways with ultimate activation of $\mathrm{C} 3$, which then controls the final common pathway. The three pathways include the classical pathway (antibody binding), lectin pathway (carbohydrate and oxidative stress activation), and the alternative pathway (microbial pathogens and cellular debris). Activation of the complement pathway induces lysis of the target cell. CFH is a regulatory protein of the complement cascade and has a significant role in AMD development and progression. $\mathrm{CFH}$ is synthesised in the $\mathrm{RPE}$ and inhibits the activation of C3. It is also able to interact with cell surface receptors to confer a resistance to damage by activated complement. Identification of polymorphism in the $\mathrm{CFH}$ gene has provided evidence of a risk allele. ${ }^{36-38}$ Long term exposure of the RPE to oxidised POS as well as influence from cytokines IL-6 and TNF- $\alpha$ downregulates the production of $\mathrm{CFH}^{39}$

Other genetic studies have found the association with factor $\mathrm{B} / \mathrm{C} 2$ and $\mathrm{C} 3$ polymorphisms to influence the risk of AMD development. ${ }^{40}$

It remains unclear how the genetic polymorphisms lead to AMD. However, it is becoming clear that these genetic polymorphisms can increase the risk of systemic complement activation. The latter is important in the pathogenesis of AMD, probably by alternation of the RPE cellular response to injury. Hence, the effect is indirect. We have previously shown that there is systemic complement activation in AMD patients indirectly measured by a degradation product of $\mathrm{C} 3$ but they are not dependant upon the $\mathrm{CFH}$ genotypes, this suggests that CFH 'at risk' polymorphisms increase the risk but secondary (either genetic or environmental) factors are required to increase systemic complement activation, which in turn lead to AMD.

\section{Inflammation biomarkers}

CRP is a biomarker believed to be a transient marker of systemic inflammation, recently it was found to be an independent risk factor for atherosclerotic disease once active infection and known systemic inflammatory disease such as rheumatic arthritis is excluded. ${ }^{41}$ As mentioned previously, one can consider the Bruch's membrane as a specialised blood vessel wall, ${ }^{10}$ it is not surprising that elevated CRP level is also associated with AMD. ${ }^{42}$

\section{AMD prevention strategies based on inflammation prevention}

There are several companies working on complement deactivation, mostly from the field of allergy. Unfortunately none of them are likely to be used in AMD, due to several limitations regarding their use; which is beyond the scope of this review. Conceptually, to reduce the effect of systemic complement activation, first we have to be able to identify one or more biomarkers, which can be used to reliably predict AMD progression. Furthermore, a reduction of the level of these biomarkers can slow or halt AMD progression. This concept is not foreign to ophthalmologists, as we routinely use intraocular pressure as a surrogate marker for visual field loss in glaucoma progression.

The use of statins has been associated with the reduction of risk of AMD. The effect is complex with comorbidities and confronting factors making it difficult to interpret the epidemiological data. Randomised controlled trials are also difficult to perform.

Nonetheless, the beneficial effect of statins in strokes is above and beyond the effect of cholesterol lowering effect, $^{42}$ it is shown in animal and human models that statins have an anti-inflammatory effect. ${ }^{43}$ This effect might be the key to AMD risk reduction. So it is reasonable for the ophthalmologists to support the use of statins in those with some increased risk of stroke as well as those with some increased risk of AMD. However, the use of statins for AMD alone cannot be recommended on current data.

\section{CNV treatment strategies based on immuno-modulation}

\section{Immunosuppression}

Many studies have identified inflammation as one of the contributory factors in AMD pathogenesis. 
Anti-inflammatory medication such as steroid, is widely used as an adjunctive treatment modality in AMD. It is likely that the effect is to control the inflammatory process of PDT. Nonetheless, it is unclear whether it has additional effect in combination with Lucentis. Depot injection in the form of Posurdex has been tested more extensively in macular oedema due to diabetes and vascular occlusion with significant improvement in oedema and visual acuity. It is unclear whether a combination of Lucentis and Posurdex might be beneficial in terms of either visual outcome or reduction of the number of Lucentis injections.

Other forms of immunosuppressants currently under investigations including the use of infliximab, sirolimus, and daclizumab. Patients with early AMD are treated and evaluated for its potential for reducing the risk of progression. Patients are randomised to either intravenous infliximab or daclizumab on a monthly basis or on alternate days of oral sirulimus.

\section{Choroidal neovascularisation: hypoxia and angiogenesis}

Angiogenesis is involved in the formation of choroidal neovascularisaton in exudative AMD, whether it is in response to hypoxia or inflammation is debatable. It is likely both play a part in the disease process. This dynamic process is regulated by vascular endothelial growth factor (VEGF-A) of which there are several different isoforms. The VEGF 121 and 165 are proven to be upregulated in the RPE, outer nuclear layers, and in CNV in hypoxic conditions. ${ }^{44}$ Increased VEGF mRNA expression in response to hypoxia is regulated by growth factors such as epidermal growth factor, TGF- $\alpha$, and TGF- $\beta$, PDGF and insulin like growth factor. In AMD, thickening of Bruch's membrane by lipid and induction of fibrosis from photosensitization ${ }^{45}$ leads to reduced diffusion of oxygen and nutrients across from the choroid to the RPE, hence producing relative chronic hypoxic conditions.

\section{Treatment of hypoxia and angiogenisis}

The mainstay of treatments provides mechanisms by which the expression, binding and response to VEGF can be altered. Other treatments include treatment of the effect of the VEGF expression.

\section{VEGF and VEGFR expression}

To counteract VEGF upregulation, treatments aimed to block VEGF expression with the use of small segments of double-stranded RNA - small interfering RNA (siRNA) to degrade target mRNA thus silencing the expression of
VEGF. The use of siRNA is being investigated via bevasiranib (Opoko Health, Miami) formerly called Cand5. The phase II CARE trial - (Cand5 Anti-VEGF RNAi Evaluation) has shown some results. Phase III trials COBALT (combining bevasiranib and Lucentis trial) is currently enrolling. This is a comparison of Lucentis alone compared to 8 or 12 weekly injections following an initial loading dose of Lucentis. Similarly, siRNA can be used to block the production of VEGFReceptor 1 (VEGFR1), this was investigated by the company SIRAN with sirna 27, but the company was taken over by Merck and they are codeveloping this molecule with Allergan.

\section{Binding VEGF}

The mainstay of treatment for exudative AMD is the use of antibodies (Avastin), antibody fragments (Lucentis) or aptamers (Macugen) to bind directly to the VEGF molecule. These anti-VEGF treatments have been extensively discussed elsewhere and so will not form part of this review.

\section{VEGF trap-eye}

A further method of blocking VEGF is to use a VEGF trap. This is a designer fusion molecule combining part of the binding site of both VEGF receptors (VEGFR1 and VEGFR2). It is able to bind to VEGF and PIGF to prevent initiation of its action. It has a very high affinity for VEGF binding as compared to Lucentis. Initially systemic VEGF trap was used, however, due to the significant adverse side effects in the form of hypertensive crisis this line of investigation was halted. Phase II trial of intravitreal VEGF-Trap is a multicentre randomised trial. This enrolled 150 patients with five treatment arms. Two groups received three doses of either 0.5 or $2.0 \mathrm{mg}$ of VEGF Trap-Eye over 12 weeks. The other three groups received a single dose of $0.5,2.0$, or $4.0 \mathrm{mg}$ of VEGF TrapEye. A significant reduction in central retinal thickness from baseline was seen in all groups after 12 weeks. This was associated with a significant improvement in visual acuity of 5.9 letters from baseline. VEGF Trap-Eye was generally well tolerated with no significant adverse effects. Due to the higher affinity, the drug can achieve significant blockade even at a lower dosage, suggesting that it might have a longer duration of action, translating into less frequent injection. A phase III clinical trial comparison with Lucentis is now enrolling - VIEW 1 (VEGF Trap: investigation of efficacy and safety in wet age-related macular degeneration).

\section{Blocking signal transduction}

VEGF receptor activation produces a cascade of phosphorylation of target proteins such as PI3, MAPK, 
and PKC. In the retina an increased level of $\beta$-isoforms of PKC are a target for treatment of AMD. SU5416 (Pfizer) and SU11248 (Takahashi) are selective inhibitors of VEGFR-2 tyrosine kinase, which have been shown to reduce $\mathrm{CNV}$ leakage in laser-induced $\mathrm{CNV}$ in animal models. PKC412 (Novartis) has been shown to be effective in animal models via both oral and periocular use. $^{46}$ This has also been shown to be effective in diabetic macular oedema. Ruboxistaurin mesylate, a PKC $\beta$ inhibitor, has now completed phase-III trials for diabetic macular oedema, and may be a potential treatment option in AMD. Implantation telescope ${ }^{47}$ might be useful in some patients with end-staged disease. Similarly, low vision support with modern electronic gadgets cannot be ignored.

Retanne ${ }^{\circledR}$ was previously investigated for the treatment of $\mathrm{CNV}$, it is a modified steroid but has antiangiogenic effects at the level of intracellular kinase pathway. The clinical trial results were not significantly better than PDT but has the convenience of using a modified subtenon depot injection every 6 months. It is licensed in Australia but failed to obtain marketing approval in United States and the European Union. However, there is a large-scale prospective randomised controlled clinical trial in risk reduction using Retanne for high-risk fellow eyes of CNV patients. Although recruitment is now completed, no results are currently in the public domain.

\section{Other anti-angiogenic options}

Many treatments for angiogenesis help to prevent the formation of new blood vessels. New molecules, such as combretastatin (Oxigene), which is an analogue of colchicine are in development. This drug selectively alters the shape of the vascular endothelial cell in the new vessels causing vascular occlusion thus depriving the $\mathrm{CNV}$ of its blood supply. This molecule is at the early stages of trials for the treatment of AMD.

\section{Other considerations}

It is impossible to cover all of AMD management in a review like this, nonetheless, we should not forget the management of those patients who despite our best effort are left with poor vision. Implantation telescope ${ }^{46}$ might be useful in some patients with end-staged disease. Similarly, low vision support with modern electronic gadgets cannot be ignored.

Other surgical methods include macular translocation, RPE patch therapy with and without the use of stem cells, localised radiation therapy (Neovista), artificial retina, are potential options, however, with the advance of Lucentis, one should consider the ethical issues in performing pilot study in those who can be benefited from Lucentis.

Finally, depression is common among those with endstaged AMD, it is important for the ophthalmologists to consider the psychiatric effect on our patients; appropriate referral might also be beneficial.

\section{Conclusion}

AMD is the current leading cause of blindness in the developed world. Modern anti-VEGF therapies combining with early detection should significantly reduce blindness. Nonetheless, significant visual loss and poor reading ability remains a problem in those antiVEGF treated patients, prevention remains the key for future management of AMD. Over the past decade of AMD research, we have a clearer idea of the pathogenesis of AMD, and we are at the turning point in the development of AMD treatment strategies.

\section{References}

1 Wang JJ, Rochtchina E, Lee AJ, Chia EM, Smith W, Cumming RG et al. Ten year incidence and progression of age-related maculopathy: the blue mountains eye study. Ophthalmol 2007; 114(1): 92-98.

2 Van Leeuwen R, Klaver CC, Vingerling JR, Hofman A, De Jong PT. The risk and natural course of age-related maculopathy: follow up at $6 \frac{1}{2}$ years in the Rotterdam study. Arch Ophthalmol 2003; 121(4): 519-526.

3 Klein R, Klein BE, Knudtson MD, Meuer SM, Swift M, Gangnon RE. Fifteen year cumulative incidence of agerelated macular degeneration: the Beaver Dam Eye Study. Ophthalmol 2007; 114(2): 253-262.

4 Age-Related Eye Disease Study Research Group. A randomized, placebo controlled, clinical trial of highdose supplementation with vitamins $\mathrm{C}$ and $\mathrm{E}$, beta carotene, and zinc for age-related macular degeneration and vision loss: AREDS Report No. 8. Arch Ophthalmol 2001; 119(10): 1417-1436.

5 TAP trial Kaiser PK for the Treatment of Age-Related Macular Degeneration with Photodynamic Therapy (TAP) Study Group. Photodynamic therapy of subfoveal choroidal neovascularisation in age-related macular degeneration: five-year results of 2 randomised clinical trials with an open label extension. Graefe's Arch Clin Exp Ophthalmol 2006; 244: 1132.

6 VEGF Inhibition Study in Ocular Neovascularisation (VISION) Clinical Trial Group. Year 2 efficacy results of 2 randomised controlled clinical trials of pegaptnib for neovascular age-related macular degeneration. Ophthalmol 2006; 113: 1508.

7 Brown DM for the ANCHOR Study Group. Ranibizumab versus verteporfin for neovascular age-related macular degeneration. N Engl J Med 2006; 355: 1432.

8 Rosenfeld PJ for the MARINA Study Group. Ranibizumab for neovascular age-related macular degeneration. $\mathrm{N} \mathrm{Engl} \mathrm{J}$ Med 2006; 355: 1419.

9 Chong NHV, Keonin J, Luthert P, Frennesson C, Weingeist $\mathrm{D}$, Wolf $\mathrm{R}$ et al. Decreased thickness and integrity of the 
macular elastic layer of Bruch's membrane correspond to the distribution of lesions associated with age-related macular degeneration. Am J Pathol 2005; 166(1): 241-251.

10 Sivapradad S, Bailey TA, Chong NHV. Bruch's membrane and the vascular intima: is there a common basis for agerelated changes and disease? Clin Experiment Ophthalmol 2005; 33(5): 518-523. Review.

11 Susuki M, Kamei M, Itabe H, Yoneda K, Bando H, Kume N et al. Oxidized phospholipids in the macula increase with age and in eyes with age-related macular degeneration. Mol Vis 2007; 13: 772-778.

12 Sies H. Oxidative stress and antioxidants. Exp Physiol 1997; 82: 291-295.

13 Winkler B, Boulton ME, Gottsch JD, Sternberg P. Oxidative damage and age-related macular degeneration. $\mathrm{Mol}$ vis 1999; 5: 32.

14 Kaemmerer E, Schutt F, Krohne TU, Holtz FG, Kopitz J. Effects of lipid peroxidation-related protein modifications on RPE lysosomal functions and POS phagocytosis. Invest Ophthalmol Vis Sci 2007; 48(3): 1342-1347.

15 Khan JC, Shahid H, Thurlby DA, Bradley M, Clayton DG, Moore AT et al. Genetic Factors in AMD study. Age-related macular degeneration and sun exposure, iris colour and skin sensitivity to sunlight. $\mathrm{Br} J$ Ophthalmol 2006; 90(1): 29-32.

16 Mitchell P, Smith W, Wang JJ. Iris color, skin sun sensitivity, and age-related maculopathy. The Blue Mountains Eye Study. Ophthalmol 1998; 105(8): 1359-1363.

17 Margrain TH, Boulton M, Marshall J, Sliney DH. Do blue light filters confer protection against age-related macular degeneration? Prog Retin Eye Res 2004; 23: 523-531.

18 Decanini A, Norgaard C, Feng X, Ferrington D, Olsen T. Changes in redox proteins of the retinal pigment epithelium in age-related macular degeneration. Am J Ophthalmol 2007; 143: 607-615.

19 O'Connell E, Neelam K, Nolan J, Eong K, Beatty S. Macular carotenoids and age-related maculopathy. Ann Acad Med Singapore 2006; 35: 821-830.

20 Thornton J, Edwards R, Mitchell P, Harrison RA, Buchan I, Kelly SP. Smoking and age-related macular degeneration: a review of association. Eye 2005; 19: 935-944.

21 Evans JR, Fletcher AE, Wormald RP. 28000 cases of agerelated macular degeneration causing visual loss in people aged 75 years and above in the United Kingdom may be attributable to smoking. Br J Ophthalmol 2005; 89: 550-553.

22 Tomany SC, Wang JJ, Van Leeuwen R, Klein R, Mitchell P, Vingerling JR et al. Risk factors for incident age-related macular degeneration: pooled findings from 3 continents. Ophthalmol 2004; 111: 1280-1287.

23 Despriet DD, Klaver CC, Witteman JC, Bergen AA, Kardys I, de Maat MP et al. Complement factor $\mathrm{H}$ polymorphism, complement activators, and risk of age-related macular degeneration. JAMA 2006; 296: 301-309.

24 Bernhard D, Wang XL. Smoking, oxidative stress and cardiovascular diseases - do anti-oxidative therapies fail? Curr Med Chem 2007; 14(16): 1703-1712. Review.

25 Nolan JM, Stack J, O'Donovan O, Loane E, Beatty S. Risk factors for age-related maculopathy are associated with a relative lack of macular pigment? Exp Eye Res 2007; 84: 61-74.

26 Hammond Jr BR, Wooten BR, Snodderly DM. Cigarette smoking and retinal carotenoids: implications for agerelated macular degeneration. Vision Res 1996; 36: 3003-3009.
27 Sastry BV, Hemontolor ME. Influence of nicotine and cotinine on retinal phospholipase A2 and its significance to macular function. J Ocul Pharmacol Ther 1998; 14: 447-458.

28 Espinosa-Heidmann DG, Suner IJ, Catanuto P, Hernandez EP, Marin-Castano ME, Cousins SW. Cigarette smokerelated oxidants and the development of sub-RPE deposits in an experimental animal model of dry age-related macular degeneration. Invest Ophthalmol Vis Sci 2006; 47: 729-737.

29 Krinsky NI, Landrum JT, Bone RA. Biologic mechanisms of the protective role of lutein and zeaxanthin in the eye. Annu Rev Nutr 2003; 23: 171-201.

30 Kanis MJ, Berendschot TT, Van Norren D. Influence of macular pigment and melanin on incident early AMD in a white population. Garaefs Arch Clin Exp Ophthalmol 2007; 245(6): 767-773.

31 Mares-Pearlman JA, Millen AE, Ficek TL, Hankinson SE. The body of evidence to support a protective role for lutein and zeaxanthin in delaying chronic disease. Overview. J Nutr 2002; 132(3): 518S-524S.

32 Bone RA, Landrum JT, Cao Y, Howard AN, AlvarezCalderon F. Macular pigment response to a supplement containing meso-zeaxanthin, lutein and zeaxanthin. Nutrition \& Metabolism 2007; 4: 12.

33 Trieschmann M, Beatty S, Nolan J, Hense H, Heimes B, Austermann $\mathrm{U}$ et al. Changes in macular pigment optical density and serum concentrations of its constituent carotenoids following supplemental lutein and zeaxanthin: The LUNA Study? Exp Eye Res 2007; 84(4): 718-728.

34 Delcourt C, Carriè re I, Delage M, Barberger-Gateau P, Schalch W, the POLA Study Group. Plasma lutein and zeaxanthin and other carotenoids as modifiable risk factors for age-related maculopathy and cataract: The POLA Study. Invest Ophthalmol Vis Sci 2006; 47: 2329-2335.

35 Mainster MA. Violet and blue light blocking intraocular lenses: photoprotection versus photoreception. $\mathrm{Br} J$ Ophthalmol 2006; 90(6): 784-792.

36 Klein RJ, Zeiss C, Chew EY, Tsai JY, Sackler RS, Haynes C et al. Complement factor $\mathrm{H}$ polymorphism in age-related macular degeneration. Science 2005; 308(5720): 385-389.

37 Haines JL, Hauser MA, Schmidt S, Scott WK, Olson LM, Gallins $\mathrm{P}$ et al. Complement factor $\mathrm{H}$ variant increases the risk of age-related macular degeneration. Science 2005; 308(5720): 419-421.

38 Hageman GS, Anderson DH, Johnson LV, Hancox LS, Taiber AJ, Hardisty LI et al. A common haplotype in the complement regulatory gene factor $\mathrm{H}$ (HF1/CFH) predisposes individuals to age-related macular degeneration. Proc Natl Acad Sci USA 2005; 102(20): 7227-7232.

39 Chen M, Forrester JV, Xu H. Synthesis of complement factor $\mathrm{H}$ by retinal pigment epithelial cells is downregulated by oxidized photoreceptor outer segments. Exp eye research 2007; 84(4): 635-645.

40 Yates JR, Sepp T, Matharu BK, Khan JC, Thurlby DA, Shahid $\mathrm{H}$ et al. Complement $\mathrm{C} 3$ variant and the risk of age-related macular degeneration. $N$ Engl J Med 2007; 357(6): 553-561.

41 Sneddon JM, George S, Rosner B, Raifi N. Progression of age-related macular degeneration: prospective assessment of C-reactive protein, interleukin 6, and other cardiovascular biomarkers. Arch Ophthalmol 2005; 123(6): 774-782.

42 Paciaroni M, Hennerici M, Angelli G, Bogousslavski J. Statins and stroke prevention. Cerebrovasc Dis 2007; 24(2-3): 170-182. 
43 Chang LT, Sun CK, Chiang CH, Wu CJ, Chua S, Yip HK. Impact of simvastatin and losartan on antiinflammatory effect: in vitro study. J Cardiovasc Pharmacol 2007; 49(1): 20-26.

44 Shima DT, Adamis AP, Ferrara N, Yeo TK, Allende R, Folkman $\mathrm{J}$ et al. Hypoxic induction of endothelial cell growth factors in retinal cells: identification and characterization of vascular endothelial growth factor (VEGF) as the mitogen. Mol Med 1995; 1(2): 182-193.

45 Gottsch JD, Pou S, Bynoe LA, Rosen GM. Hematogenous photosensitization. A mechanism for the development of age-related macular degeneration. Invest Ophthalmol Vis Sci 1990; 31: 1674-1682.

46 Saishin Y, Lima Silva R, Saishin Y, Callahan K, Schoch C, Ahlheim $\mathrm{M}$ et al. Periocular injection of microspheres containing PKC412 inhibits choroidal neovascularization in a porcine model. Invest Ophthalmol Vis Sci 2003; 44(11): 4989-4993.

47 Hudson HL, Lane SS, Heier JS, Stulting RD, Singerman L, Lichter PR et al. Implantable miniature telescope for the treatment of visual acuity loss resulting from end stage agerelated macular degeneration: 1 year results. Ophthalmol 2006; 113: 1982-2001. 